\title{
Competency of Emergency Physicians in Interpretation of Computerized Brain Tomography in Non-Traumatic Cases
}

\author{
(1) Mümine Yiğit ${ }^{1}$, (1) Mehmet Akçimen²
}

${ }^{1}$ Clinic of Emergency Medicine, Antalya Kepez Public Hospital, Antalya, Turkey

${ }^{2}$ Clinic of Emergency Medicine, Antalya Training and Research Hospital, Antalya, Turkey

\begin{abstract}
Aim: The Computerized Brain Tomography is extremely used in the diagnosis of the neurological diseases. The aim of the study was to evaluate the competency of emergency medicine physicians' in the evaluations and diagnosis of "computerized brain tomography" (CBT) of the neurological disease findings in non-traumatic cases

Materials and Methods: This study was made in the Emergency Department of a tertiary training hospital between 2015 and 2016 . The CBT images, taken for the patients were evaluated by the emergency medicine physician. The tomography interpretations, provided by the radiology physicians, were considered as the gold standardas the accurate finding, and findings of the emergency medicine physician were compared with the accurate findings.

Results: Totally 197 patients inclueded. It was found that the sensitivity and specificity were as 96\% and 88\% in the grey white or separation, sensitivity was $91 \%$ and specificity was $76 \%$ in the ventricle constriction, average sensitivity and specificity were $99 \%$ and $86 \%$ in the SAK, EDH and SDH, and $99 \%$ and $97 \%$ in the cerebral infarct, and $97 \%$ and $91 \%$ in the cerebellar infarct, respectively.

Conclusion: In our study, emergency medicine physicians were highly successful in the interpretation of the CBT of patients with nontraumatic neurological incidents and it was in an excellent harmony with the radiologists' interpretations. The success rates of interpretation of CBT may be increased with practical and theoretical comprehensive training of the emergency medicine physicians.
\end{abstract}

Keywords: Emergency physician, non-traumatic neurological disease, computerized brain tomography, radiologist, competency

\section{Introduction}

Nowadays, emergency departments are an access point for the healthcare service of the patients, whose general situations are bad and critical. The brain vascular diseases occurring with the non-traumatic reason constitute a substantial part of the patients, who applied to the emergency department with the neurological indications and findings. The brain vascular diseases occurring with the non-traumatic reasons are the second leading cause of death following the heart disease and cancer in developed countries $(1,2)$. It is also in the lead in terms of causing the death and disability among the neurological diseases in adults. It has an important place in the hospital applications and health expenses for the countries having a high socioeconomic level (1-4).
Computerized Brain Tomography (CBT) provides the rapid and reliable results in characterizing the life-threatening intracranial lesions such as intracranial hemorrhage, tumor and hydrocephaly and in a case where the emergency action is required. The usage area of CBT expanded by the technological developments for diagnosis, follow-up or research. The image quality and diagnostic accuracy rate of lesions increased by CBT (5).

CBT is frequently used in our emergency department as a substantial component of the clinical evaluation. Although many CBT studies in the emergency departments were made, the studies revealing CBT evaluation competency of emergency medicine physicians were still less. 
In our study, we aimed at researching the competency of emergency physicians in CBT evaluations for the non-traumatic cases. The aim of the study was to evaluate the competency of emergency medicine physicians' in the evaluation and diagnosis of "Computerized Brain Tomography" (CBT) of the neurological disease findings in non-traumatic cases.

\section{Materials and Methods}

This study was planned in a tertiary training hospital. An approval was taken from the ethics committee of the hospital after the study was planned.

The radiology and emergency medicine clinic trainers provided totally 4-hour training to the emergency medicine physicians about CBT evaluations, including 2-hour theoretical and 2-hour practical training.

CBT images of the patients included in the study were taken by Hitachi ${ }^{\circ}$ 16-sliced tomography equipment, found in the Emergency Tomography unit within the emergency department. CBT scans for the patients were performed by using enhanced or unenhanced methods. The study was designed in order for the emergency physician to record the findings by evaluating CBT images taken and compare the findings of patients with these accurate radiology findings.

The subjects of this study were prospectively planned by oneyear period starting from 01.03.2015 to 01.03.2016. The study included the patients at the age of 18 and over, who applied to the emergency department with the non-traumatic reasons and whose CBT was taken as a diagnostic examination during the evaluation. An informed consent was taken by the patients. The study excluded from the patients at the age of 18 and below, who had CBT images during the application, did not give a written consent and whose diagnoses were available.
CBT images taken to the patients were evaluated by emergency physician, and findings were recorded on the form prepared. The recommended findingswere indicated by the form by marking whether they were available (Table 1).

\section{Statistical Analysis}

The data were analyzed using SPSS version 23 (Statistical Package for the Social Sciences - IBM ${ }^{2}$ ).

The descriptive statistics were submitted as numbers and percentages for the categorical variables, and mean and standard deviation for the numerical variables. While the ANOVA test was used for the numerical variables in a case where the normal distribution assumption was provided for comparing the multiple independent groups, otherwise the KruskalWallis Test was used. The chi-square test statistic was used for the categorical variables. The wrong positive, wrong negative, sensitivity, specificity, Positive Predictive Value (PPV), Area under curve (AUC) and kappa coefficient were calculated for computed tomography (CT) interpretations. The confidence interval of 95\% and $p<0.05$ values were used by statistically determining the significance level.

\section{Results}

The study included 197 patients, who presented atto the emergency department of tertiary training hospital and whose computerized brain tomography (CBT) was taken as a diagnostic method.

When demographical data of the patients included in the study scope were reviewedand age ranges of the patients included in the study scope were evaluated, the age ranges were calculated asas $18-45$ for $11.7 \%$, as $46-55$ for $33 \%$ and as $<66$ for $53.3 \%$. Mean

\begin{tabular}{|l|l|}
\hline \multicolumn{2}{|l|}{ Table 1. Findings demanded to be specified by the emergency physicians in CBT evaluation } \\
\hline - Cerebral edema findings & $\bullet$ Intracranial shift existence \\
- Grey-white ore separation & $\bullet$ Arterio-venous malformation finding \\
- Extinction in sulcus & Intraparenchymal hemorrhage \\
- Constriction in ventricles & - Enlargement in lateral ventricle \\
- Subdural hematoma & - Enlargement in $3^{\text {rd }}$ ventricle \\
- Spidural hematoma & - Enlargement in $4^{\text {th }}$ ventricle \\
- Subarachnoidal hemorrhage & $\bullet$ Sinusitis findings \\
- Infarct areas & $\bullet$ Venous sinus pathology \\
- Cerebral infarct & - Hydrocephaly \\
- Cerebellar infarct & $\bullet$ Bone pathology \\
- Intracranial mass existence & \\
- Edema effect & \\
- Shift existence & \\
- Hemorrhage in mass & \\
\hline The findings in the CBT in the radiology report and the findings determined by the emergency physician were compared at the end of study. \\
CBT: Computerized brain tomography
\end{tabular}


age \pm standard deviation (SD) of the patients was calculatedas $64.69 \pm 1.13$ [minimum $(\min )=19$ and $\operatorname{maximum}(\max )=96]$.

The patients included in the study scope were females $44.2 \%$ and males $55.8 \%$. While Unenhanced CBT scan was performed for $97.5 \%$ of the patients taking part in our study, both enhanced and unenhanced CBT scans and just Enhanced CBT were performed for $1.5 \%$ and $1 \%$ of the patients, respectively.

The cerebrovascular event (one-sided strength loss at 34\% rate, consciousness change at $23.9 \%$ rate and speech disorder at $11.2 \%$ rate) was determined as an application reason to the emergency department in 136 patients (69\%), who applied to the emergency department of our hospital and were included in the study scope. A headache in 17 patients (8.6\%), dizziness in 15 patients (7.6\%), fainting in 15 patients (7.1\%), seizure in 14 patients (4.1\%) and visual disorder (1\%) were determined to follow up these complaints (Table 2).

When consciousness states of the patients were evaluated during the application for the emergency department, the Glasgow Coma Score (GCS) was determined as 15 for 136 patients (69\%), as 13-14 for 37 patients (18.8\%), as $9-12$ for 15 patients (7.6\%) and as 3-8 for nine patients (4.6\%) (Table 3).

When examination and treatment outcomes of the patients taking part in the study are evaluated, while $40.6 \%$ of 197 patients were discharged; the remaining patients were hospitalized as $33.5 \%$ for the neurology service, as $12.2 \%$ for the intensive care unit, as $2 \%$ for the internal diseases service, as $3 \%$ for the neurosurgery service, as $1.5 \%$ for the oncology service and as $1 \%$ for the infectious diseases service (Table 4).

No statistically significant difference was detected as regards evaluating the life-threatening diagnosissuch as cerebral infarct, cerebellar infarct, and intracranial mass, intracranial shift existence, and intraparenchymal hemorrhage, enlargement in the sulcus and bone pathology between the emergency medicine physician and radiologist. But, a statistically significant difference was detected for the diagnosis of the non-fatal findingssuch as arteriovenous (AV) malformation, sinusitis, venous sinus pathology and hydrocephaly (Table 5).

The sensitivity, specificity, positive predictive value (PPV) and percentage of the values were shown in Table 6 for the success of the emergency medicine physician in the first diagnosis.

\section{Discussion}

The non-traumatic brain vascular diseases come as the third leading cause of death followingthe heart disease and cancer in developed countries (1,2). Fifteen million of humans have a stroke in every year around the world. Early diagnosis and treatment of the stroke have a substantial effect on the neurological functions and survival (6). CBT is one of the effective methods, which is used to determine the fibrinolysis contraindications and provide the stroke patient to receive a fibrinolytic treatment for the early period. CBT is the most cost-effective imaging strategy to be performed in the stroke patients for whom is rapidly taken

\begin{tabular}{|l|l|l|}
\hline Table 2. Reasons for interpretation of computerized brain tomography (CBT) \\
\hline Primary complaint & $\mathbf{n = 1 9 2}$ & $\%$ \\
\hline $\begin{array}{l}\text { One-sided strength loss } \\
\text { Consciousness change } \\
\text { Speech disorder }\end{array}$ & 136 & 69 \\
\hline Headache & 17 & 8.6 \\
\hline Dizziness & 15 & 7.6 \\
\hline Fainting & 14 & 7.1 \\
\hline Seizure & 8 & 4.1 \\
\hline Visual disorder & 2 & 1 \\
\hline The data are expressed as numbers (percentages), n: Number & & \\
\hline
\end{tabular}

\begin{tabular}{|l|l|l|}
\hline \multicolumn{2}{|l|}{ Table 3. Glasgow Coma Scale (GCS) data } \\
\hline GCS & $\mathrm{n}=197$ & $\%$ \\
\hline 15 & 136 & 69 \\
\hline $13-14$ & 37 & 18.8 \\
\hline $9-12$ & 15 & 7.6 \\
\hline $3-8$ & 9 & 4.6 \\
\hline The data are expressed as numbers (percentages), n: Number & & \\
\hline
\end{tabular}


Table 4. Outcomes of the patients included in the study

\begin{tabular}{|c|c|c|c|}
\hline & & $\mathrm{n}=197$ & $\%$ \\
\hline \multirow{8}{*}{ Outcomes } & Hospitalization to intensive care unit & 24 & 12.2 \\
\hline & Hospitalization to neurology service & 66 & 33.5 \\
\hline & Referral to outside healthcare service & 10 & 5.1 \\
\hline & Hospitalization to internal diseases service & 4 & 2 \\
\hline & Hospitalization to neurosurgery service & 6 & 3 \\
\hline & Hospitalization to oncology service & 3 & 1.5 \\
\hline & Hospitalization to infectious diseases service & 2 & 1 \\
\hline & Hospitalization to chest diseases service & 1 & 0.5 \\
\hline
\end{tabular}

after bringing to the emergency department; however, it is not sensitive to the hemorrhages occurring before (7).

Although there are CT devices in many hospitals, not many sufficient radiologistsare found to interpret CT images. Moreover, there is not an interpretation system yet, rather than the physicians' interpretations in the evaluation of CT images (8). Hunter et al. (8) stated that CT images may only be interpreted by the radiologists working in developed medicine institutions and university hospitals, in their studies referring to the radiology support in the emergency departments (8). Torreggiani et al. (9) specified that the radiologist interpretation may not be simultaneously possible and the radiologist interpretation may be only performed 48 hours later, in their studies referring to the investigation of emergency radiology in Canada. Therefore, the emergency department physicians complain about the deficiency in current interpretation system.

The incidence rate of neurological diseases is observed to increase with age. The vascular elasticity decreases and its resistance increases in increasing age, and therefore, the frequency of many diseases such as hypertension, ischemic stroke, and hemorrhagic stroke increases. The patients' ages were found as $18-45$ at $11.7 \%$, as $46-55$ at $33 \%$ and as $<66$ at $53.3 \%$; when age ranges of the patients were evaluated by the study performed by us. The patients mean ages \pm SD were calculated as $64.69 \pm 1.13(\min =19$ and $\max =96)$. The similar results were obtained by the study performed by Ariesen et al. (10) in which they researched the intracranial hemorrhage risk factors of the general population, the study performed by Soriano-Tárraga et al. (11) in which they performed related to the age and stroke, and many studies performed in this respect. Çalışkan et al. (12) determined the mean age of totally 289 patients as 51.8 years ( $\min =18$ and $\max =87$ ), including 142 males and 147 females, who presented to the emergency department of our hospitaldue to splitting headache reason and whose CT and CTA were taken by the pre-diagnosis of subarachnoid hemorrhage (SAH) and aneurysm.

The cerebro vascular diseases (one-sided strength loss, consciousness change and speech disorder) was determined as an application reason for the emergency department with the highest rate of the patients, who applied to the emergency department of our hospital and were included in the study. A headache, dizziness, fainting, seizure and visual disorder followed up these complaints. Calıșkan et al. (12) determined in their study that the patients had the complaints such as such as splitting headache and as well as vomiting, confusion, consciousness change and convulsion.

Al Reesi et al. (13) revealed in their study that the cerebrovasculary event (CVE) (22\%) and headache (21\%) were the most frequent CBT scan indications (excluding trauma). Jamal et al. (14) specified in their study that the CVE (27\%), headache (14.5\%) and suspicious cases (12\%) were the non-traumatic CBT scan indications.

Decreasing the grey-white ore separation is one of the cerebral ischemia indications in unenhanced $\mathrm{CT}$, taken in the first a few hours following starting of the symptoms. This situation reveals itself as a certain decrease in the basal ganglia nuclei or as an intermingling density of the white ore and cortex, taking part under the insula and over the convexities (1). We determined in our study that the emergency physicians successfully diagnosed disruption of the grey-white ore separation, with $88 \%$ sensitivity and $96 \%$ specificity rates, respectively.

Another finding of the cerebral ischemia is the sulcal extinction. If these findings become rapidly evident, the degree of ischemia becomes splitting, too. However, diagnosis ability of the observers varies while determining these early findings and depends on the dimension of the infarct, severity of the ischemia and 


\begin{tabular}{|c|c|c|c|c|c|}
\hline & & \multicolumn{2}{|c|}{ Emergency physician } & \multicolumn{2}{|c|}{ Radiologist } \\
\hline & & $n=197$ & $\%$ & $n=197$ & $\%$ \\
\hline \multirow{2}{*}{ Cerebral infarct* } & Yes & 61 & 31.0 & 77 & 39.1 \\
\hline & No & 136 & 69.0 & 120 & 60.9 \\
\hline \multirow{2}{*}{ Cerebellar infarct* } & Yes & 1 & 1.5 & 5 & 2.5 \\
\hline & No & 196 & 99.5 & 192 & 97.5 \\
\hline \multirow{3}{*}{ Intracranial mass* } & Yes & 3 & 1.5 & 4 & 2.0 \\
\hline & No & 188 & 95.4 & 187 & 94.9 \\
\hline & Edema effect & 6 & 3.0 & 6 & 3.0 \\
\hline \multirow{2}{*}{ Intracranial shift existence* } & Yes & 7 & 3.6 & 6 & 3.0 \\
\hline & No & 190 & 96.4 & 191 & 97.0 \\
\hline \multirow{2}{*}{ AV malformation ${ }^{* *}$} & Yes & 4 & 2.0 & 5 & 2.5 \\
\hline & No & 193 & 98.0 & 192 & 97.5 \\
\hline \multirow{4}{*}{ Intraparenchymal hemorrhage* } & Yes & 10 & 5.1 & 10 & 5.1 \\
\hline & No & 179 & 90.9 & 178 & 90.4 \\
\hline & $\begin{array}{l}\text { Enlargement in lateral } \\
\text { ventricle }\end{array}$ & 6 & 3.0 & 7 & 3.6 \\
\hline & Enlargement in all ventricles & 2 & 1.0 & 2 & 1.0 \\
\hline \multirow{2}{*}{ Sinusitis findings** } & Yes & 10 & 5.1 & 28 & 14.2 \\
\hline & No & 187 & 94.9 & 169 & 85.8 \\
\hline \multirow{2}{*}{ Venous sinus pathology** } & Yes & 0 & 0 & 1 & 0.5 \\
\hline & No & 197 & 100 & 196 & 99.5 \\
\hline \multirow{2}{*}{ Hydrocephaly** } & Yes & 0 & 0 & 1 & 0,5 \\
\hline & No & 197 & 100 & 196 & 99.5 \\
\hline \multirow{2}{*}{ Bone pathology* } & Yes & 3 & 1.5 & 4 & 2.0 \\
\hline & No & 194 & 98.5 & 193 & 98.0 \\
\hline \multirow{2}{*}{ Enlargement in sulcus* } & Yes & 24 & 12.2 & 60 & 30.5 \\
\hline & No & 173 & 87.8 & 137 & 69.5 \\
\hline \multirow{2}{*}{ Other findings* } & Yes & 2 & 1.0 & 7 & 3.6 \\
\hline & No & 195 & 99.0 & 190 & 96.4 \\
\hline
\end{tabular}

appearance of the indications with imaging (1). We determined in our study that the emergency physicians successfully diagnosed extinction in the sulcus, with $76 \%$ sensitivity and $91 \%$ specificity rates, respectively.

The emergency physician mostly makes management plan for the patient by interpreting the brain CT images, without waiting for the radiologist interpretation in many emergency departments. Therefore, the accuracy of an interpretation made by the emergency physician is very important. Dolatabadi et al. (15) determined sensitivity and specificity interpretations of the emergency physicians as $86.5 \%$ and $81.4 \%$, respectively; and they found thepositive predictive value (PPV) as 6.9\%, negative predictive value (NPV) as $86.9 \%$, positive likelihood ratio (PLR) as $4.6 \%$ and negative likelihood ratio (NLR) as $0.16 \%$, in a study performed by them by considering the radiology interpretation as a standard for the patients, who applied to the emergency department and whose brain CT was taken in the diagnosis phase (15). Khan et al. (16) revealed that the emergency department physicians found the sensitivity as $87.14 \%$, the specificity as $12.86 \%$ and the kappa value as $0.64 \%$ in interpreting the brain $\mathrm{CT}$, in the study performed by them referring to researching the evaluation of unenhanced brain tomography taken in the emergency department. Moreover, Harding et al. (17) specified that the emergency department physicians found the specificity as $98 \%$ in interpreting the brain $\mathrm{CT}$, in the study performed by 


\begin{tabular}{|c|c|c|c|c|c|}
\hline & \multicolumn{5}{|c|}{ Competency of the emergency physicians } \\
\hline & Specificity & Sensitivity & NPV & PPV & $\%$ \\
\hline Grey-white ore separation & 96.0 & 88.0 & 99.0 & 67 & 88 \\
\hline Extinction in sulcus & 91.0 & 76.0 & 98.0 & 69 & 90 \\
\hline Constriction in ventricle & 96.0 & 89.0 & 99.0 & 71 & 86 \\
\hline Subarachnoidal hemorrhage & 97.0 & 86.0 & 97.0 & 92 & 90 \\
\hline Subdural hematoma & 99.0 & 89.0 & 100.0 & 100 & 87 \\
\hline Cerebral infarct & 99.0 & 97.0 & 100.0 & 93 & 89 \\
\hline Cerebellar infarct & 97.0 & 91.0 & 100.0 & 69 & 84 \\
\hline Intracranial mass & 98.0 & 85.0 & 99.0 & 64 & 74 \\
\hline Intracranial shift existence & 94.0 & 84.0 & 98.0 & 73 & 82 \\
\hline AV malformation & 96.0 & 89.0 & 100.0 & 79 & 94 \\
\hline Intraparenchymal hemorrhage & 99.0 & 98.0 & 100.0 & 72 & 90 \\
\hline Sinusitis findings & 99.0 & 98.0 & 99.0 & 75 & 86 \\
\hline Venous sinus pathology & 95.0 & 91.0 & 99.0 & 80 & 71 \\
\hline Hydrocephaly & 99.0 & 96.0 & 98.0 & 95 & 84 \\
\hline Bone pathology & 97.0 & 84.0 & 92.0 & 64 & 86 \\
\hline Enlargement in sulcus & 92.0 & 75.0 & 90.0 & 62 & 72 \\
\hline Other findings & 89.0 & 92.0 & 89.0 & 78 & 88 \\
\hline
\end{tabular}

them referring to $\mathrm{CT}$ interpretation of the trauma patients having a SAH suspicion. In our study, an excellent harmony was found by diagnosis and brain CT interpretation of the emergency medicine physician. In addition, in our study, the sensitivity and specificity were found as $96 \%$ and $88 \%$, respectively in the grey-white ore separation, while the sensitivity was $91 \%$ and the specificity was $76 \%$ in the ventricle constriction. The mean sensitivity and specificity were found as $99 \%$ and $86 \%$, respectively in the SAH, EDH and SDH; while they were $99 \%$ and $97 \%$ in the cerebral infarct and $\% 97$ and \%91 in the cerebellar infarct, respectively. Moreover, the main sensitivity and specificity determined to vary between 89\%-99\% and 75\%-98\%, respectively in the diseases such as intracranial mass, intracranial shift existence, AV malformation, intraparenchymal hemorrhage, sinusitis findings, venous sinus pathology, hydrocephaly, bone pathology and enlargement in the sulcus.

The kappa value was found to be 0.83 in a study performed by Al Reesi et al. (13) referring to comparing the diagnosis harmony between the emergency physicians and neuroradiologist. In the same study, they revealed that the results arising from the wrong interpretation of $\mathrm{C} T$ by the emergency medicine physicians rarely occurred.

It was determined in many studies that there was a diagnostic concordance between the emergency physicians and radiologists at the rate of $60 \%-94 \%$. While this concordance was determined as $94 \%$ in the study performed by Jamal et al. (14), the kappa value was found as 0.78 .

\section{Study Limitations}

Imaging of the mass and arteriovenous malformations was determined to be constrained due to being less the number of enhanced CBT taken.

\section{Conclusion}

In our study, emergency medicine physicians were highly successful in the interpretation. The findings of vital cerebral infarct $(p=0.001)$, cerebellar infarct $(p=0.035)$, intracranial mass $(p=0.017)$, intracranial shift $(p=0.027)$ and intraparenchymal hemorrhage $(p=0.021)$ were in an excellent harmony with the radiology reports. These findings were generally found compatible with the literature. The sensitivity and specificity height constituted CBT evaluation competency of the emergency physician even in the non-vital subjects. Moreover, we thought that participation in the emergency physicians increased their competency in terms of the diagnosis during history, physical examination, follow-up and consultation periods of the patients. The success rates of interpretation of CBT may be increased with practical and theoretical comprehensive training of the emergency medicine physicians. 
In conclusion, In our study, emergency medicine physicians were highly successful in the interpretation of the CBT of patients with non-traumatic neurological incidents and it was in an excellent harmony with the radiologists' interpretations.

\section{Ethics}

Ethics Committee Approval: This study was approved by Antalya Training and Research Hospital Clinical Researches Ethics Committee (no: 62/16, date: 18.06.2015).

Informed Consent: It taken by the patients.

Peer-review: Externally peer-reviewed.

\section{Authorship Contributions}

Surgical and Medical Practices: M.A., Concept: M.A., Design: M.Y., M.K., Data Collection or Processing: M.A., M.Y., Analysis or Interpretation: M.K., Literature Search: M.A., M.Y., M.K., Writing: M.K.

Conflict of Interest: No conflict of interest was declared by the authors.

Financial Disclosure: The authors declared that this study received no financial support.

\section{References}

1. Stephen JXM, Werring DJ. Stroke: causes and clinical features. Medicine. 2020;48:561-6.

2. Miller CM. Stroke Epidemiology. In: Lapchak P., Yang GY. (eds) Translational Research in Stroke. Translational Medicine Research. Springer, Singapore, 2017 Available at: https://doi.org/10.1007/978-981-10-5804-2_3

3. Thrift AG, Howard G, Cadilhac DA, Howard VJ, Rothwell PM, Thayabaranathan T, et al. Global stroke statistics: An update of mortality data from countries using a broad code of "cerebrovascular diseases. Int J Stroke. 2017;12:796801.
4. Poorthuis MHF, Algra AM, Algra A, Kappelle LJ, Kljin CJM. Female-and malespecific risk factors for stroke: a systematic review and meta-analysis. JAMA Neurol. 2017;74:75-81.

5. Paterson A, Frush DP. Dose reduction in paediatric MDCT: general principles. Clin Radiol. 2007;62:507-17.

6. Singletary EM, Zideman DA, Bendall JC, Berry DC, Borra V, Carlson JN, et al. 2020 international consensus on first aid science with treatment recommendations. Circulation. 2020;142(suppl1):S284-S334.

7. Li H, Yan L, Li MH, Li YD, Tan HQ, Gu BX, et al. Evaluation of intracranial aneurysms with high-resolution MR angiography using singleartery highlighting technique: correlation with digital subtraction angiography. Radiol Med. 2013;118:1379-87.

8. Hunter TB, Krupinski EA, Hunt KR, Erly WK. Emergency department coverage by academic departments of radiology. Acad Radiol. 2000;7:165-70.

9. Torreggiani WC, Nicolaou S, Lyburn I, Harrsi A, Buckley AR. Emergency radiology in Canada: a national survey. Can Assoc Radiol J. 2002;53:160-7.

10. Ariesen MJ,ClausSP, RinkelGJE,AlgraA. Riskfactorsforintracerebral hemorrhage in the general population: a systematic review. Stroke. 2003;34:2060-5.

11. Soriano-Tárraga C, Giralt-Steinhauer E, Mola-Caminal M, Ois A, RodríguezCampello A, Cuadrado-Godia E, et al. Biological age is a predictor of mortality in ischemic stroke. Nature. 2018;8:1-8.

12. Çalışkan, Emine, Dilek Öncel. Şiddetli baş ağrısı nedeniyle başvuran hastalarda kranial BT anjiyografide anevrizma ve subaraknoid kanama saptanma oranı [Article in Turkish]. Tepecik Eğit Hast Derg. 2015;25:101-5.

13. Al-Reesi A, Stiell IG, Al-Zadjali N, Cwinn AA. Comparison of CT head interpretation between emergency physicians and neuroradiologists. Eur J Emerg Med. 2010;17:280-2.

14. Jamal K, Mandel L, Jamal L, Gilani S. 'Out of hours' adult CT head interpretation by senior emergency department staff following an intensive teaching session: a prospective blinded pilot study of 405 patients. Emerg Med J. 2014;31:467-70.

15. Dolatabadi AA, Baratloo A, Rouhipour A, Abdalvand A, Hatamabadi $\mathrm{H}$, Forouzanfar M, et al. Interpretation of computed tomography of the head: emergency physicians versus radiologists. Trauma Mon. 2013;18:86-9.

16. Khan A, Qashqari S, Al-Ali AA. Accuracy of non-contrast CT brain interpretation by emergency physicians: a cohort study. Pak J Med Sci. 2013;29:549-53.

17. Harding J, Craig M, Jakeman N, Young R, Jabarin C, Kendall J. Emergency physician interpretation of head CT in trauma and suspected subarachnoid haemorrhage-is it viable? An audit of current practice. Emerg Med J. 2010;27:116-20. 